\title{
Parameter Optimization in Voltage Pulse Plethysmography
}

\author{
M. Melinscak \\ Polytechnic of Karlovac, Karlovac, Croatia
}

Abstract - Measurement optimization is being studied when short voltage pulses are stimulating bio-tissue and the transient process is sampled in order to measure the tissue volume changes. The measurement sensitivity depends on the ratio of the sampling instant to the time constant of the transient process, $(T / \tau)$ and on the ratio of the current sensing resistance to the resistance of the electrode-skin interface $\left(R_{0} / R_{S X}\right)$. With variations of $R_{0} / R_{S X}$ and $T / \tau$ the sensitivity changes from negative to positive values, while it equals zero for certain $\boldsymbol{R}_{0} / \boldsymbol{R}_{S X}$ and $T / \tau$ ratios. The sensitivity is greater when positive while than negative but it depends on $T / \tau$ and $R_{0} / R_{S X}$. For negative sensitivity, $T$ and $R_{0}$ can be chosen to maximize the sensitivity and minimize its variations.

Keywords - voltage pulse plethysmography, electrode tissue interface, tissue resistance variation

\section{INTRODUCTION}

Plethysmography is a method of measuring volume changes in bio-tissue. Impedance plethysmography is most often applied in measuring volume changes due to blood pulsation (Fig. 1) or respiration (Fig. 2) whereby the biotissue resistance changes. In this study the voltage pulse plethysmography is being examined. Short voltage pulses activate the bio-tissue and the characteristics of the transient process are measured (Fig. 3, Fig. 4).

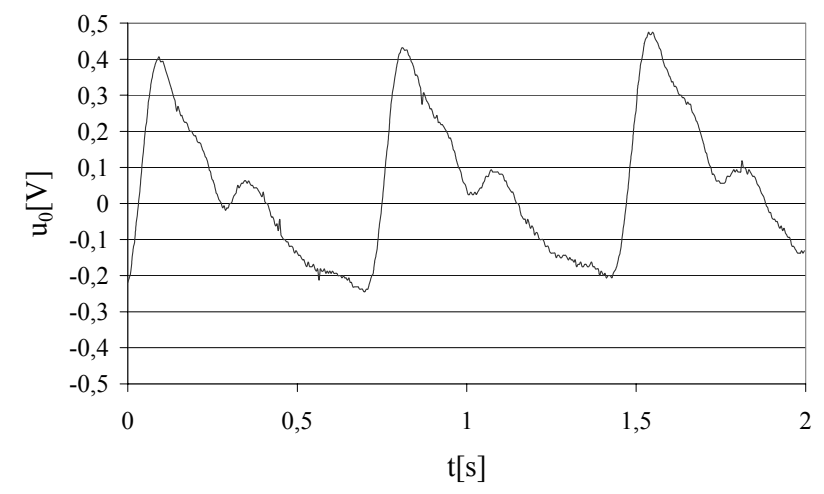

Fig. 1 The output voltage for blood pulsation in the arteries [1]

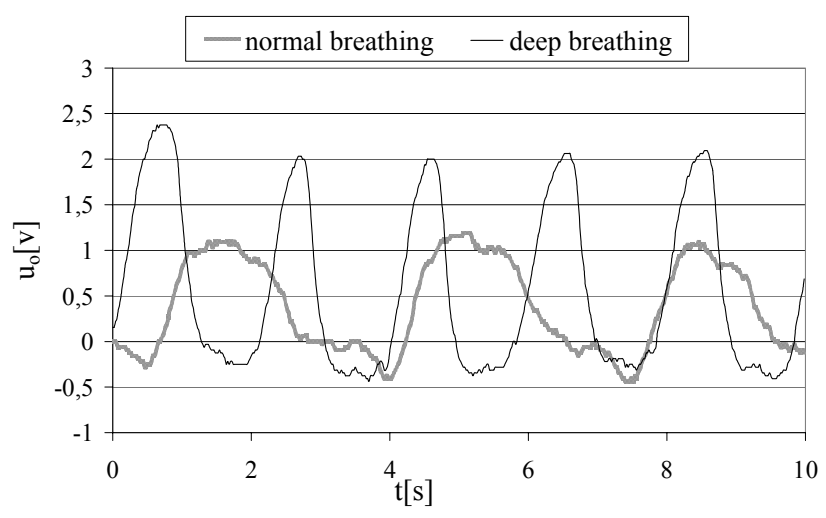

Fig. 2 The output voltage for normal and deep breathing respiration [1]

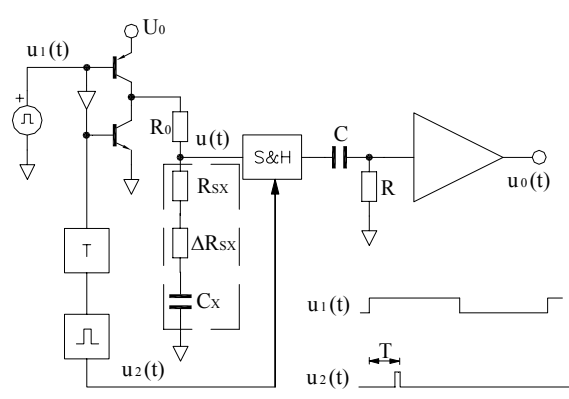

Fig. 3 Block diagram of voltage pulse plethysmography

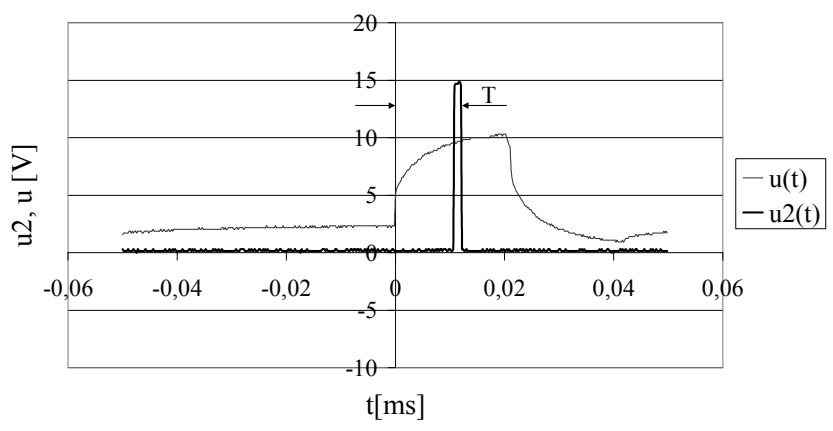

Fig. 4 Characteristic waveforms of voltage pulse plethysmography as in Fig. 3 
The electrode-skin interface can be modeled with a fiveelement circuit that can be simplified to a three-element circuit due to the circuit symmetry (Fig. 5) [2]. The impedance of the electrode-skin interface is frequency dependent [3]-[5] and at high frequencies [2], [6] which are applied in pulse plethysmography, can be replaced by a series RC-circuit (Fig. 5), where the variable resistance, $\Delta R_{S X}$ is added in series with $R_{S X}$ simulating the resistance change due to the change of the bio-tissue volume. This resistance change is rather small, typically $0.1-0.5 \%$ [6], [7].

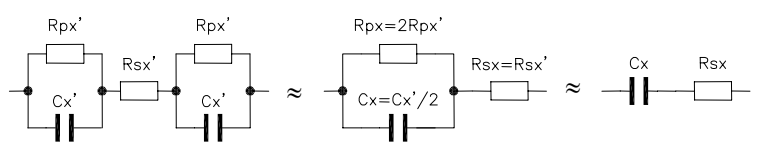

Fig. 5 Equivalent circuits for electrode-skin interface [2]

\section{OPTIMIZATION METHODS}

Fig.3 shows the block diagram of the voltage pulse plethysmography. One electrode of the circuit is grounded while voltage sample is taken on the other electrode. The analysis is performed for this implementation. The other possible implementation is when the current sampling resistor $R_{0}$ is grounded. The analysis for this implementation is very similar describing in fact the same transient process. A simple analysis results in the expression for voltage $u(t)$ :

$u(t)=U_{0} \cdot\left(1-\frac{y}{1+y+\delta R} \cdot e^{-\frac{x}{1+y+\delta R}}\right)$

where $y=R_{0} / R_{S X}, \delta R=\Delta R_{S X} / R_{S X}, x=T / \tau, \tau=R_{S X} C_{X}$.

The voltage variation with constant current sampling resistance $\mathrm{R}_{0}$ and $\Delta R_{S X} / R_{S X}$ as a parameter is shown in Fig. 6 . It is evident that all curves intersect at one point, that is, the measurement sensitivity equals zero for certain $T / \tau$ ratio with fixed $R_{0} / R_{S X}$ as a parameter. The relative measurement sensitivity is defined as the variation of the sampled voltage variation $\mathrm{d} u(t=T)$ normalized to the impulse amplitude $U_{0}$, relative to the relative change of the bio-tissue resistance $R_{S X}$ :

$S=\left(d u / U_{0}\right) /(d R / R)=-\frac{y}{(y+1)^{2}} \cdot\left(\frac{x}{1+y}-1\right) \cdot e^{-\frac{x}{1+y}}$
The measurement sensitivity depends on the voltage sampling instant, $T$ and resistance of the current sampling resistor. Dependence of the sensitivity with $\mathrm{y}=R_{0} / R_{S X}$ as a parameter is in Fig. 7 and with $x=T / \tau$ as a parameter is in Fig. 8.

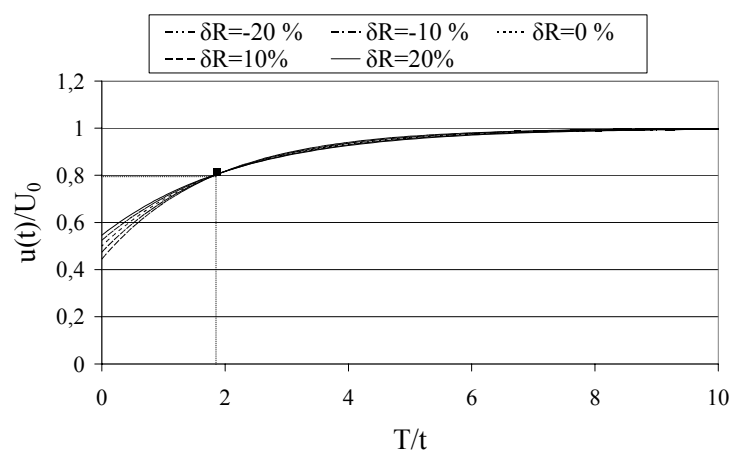

Fig. 6 Voltage dependence $u(t) / U_{0}$ with constant sampling resistance $R_{0}$ and $y=1$

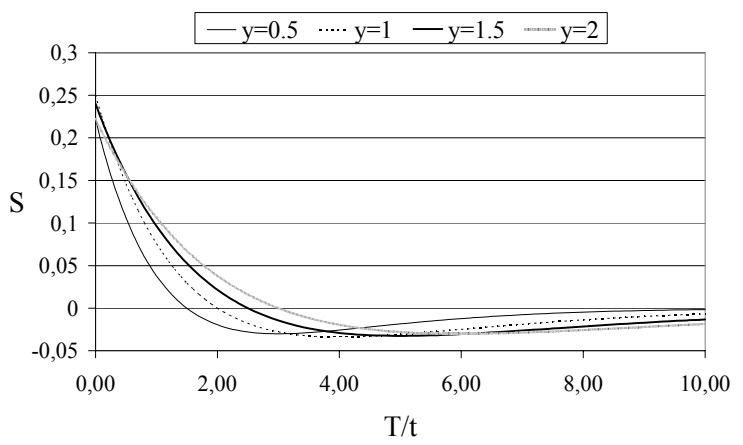

Fig. 7 Measurement sensitivity $S$ with $y=R_{0} / R_{\mathrm{SX}}$ as a parameter

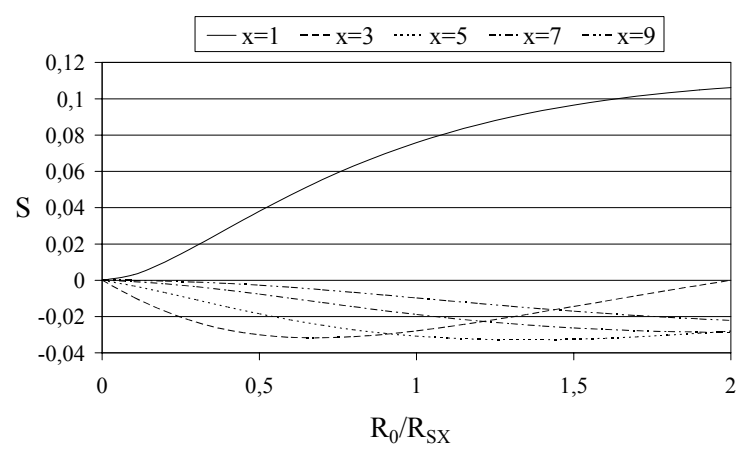

Fig. 8 Measurement sensitivity $S$ with $x=T / \tau$ as a parameter 
In Fig. 9 is a surface plot of measurement sensitivity $S$ depending on $T / \tau$ and $R_{0} / R_{S X}$.

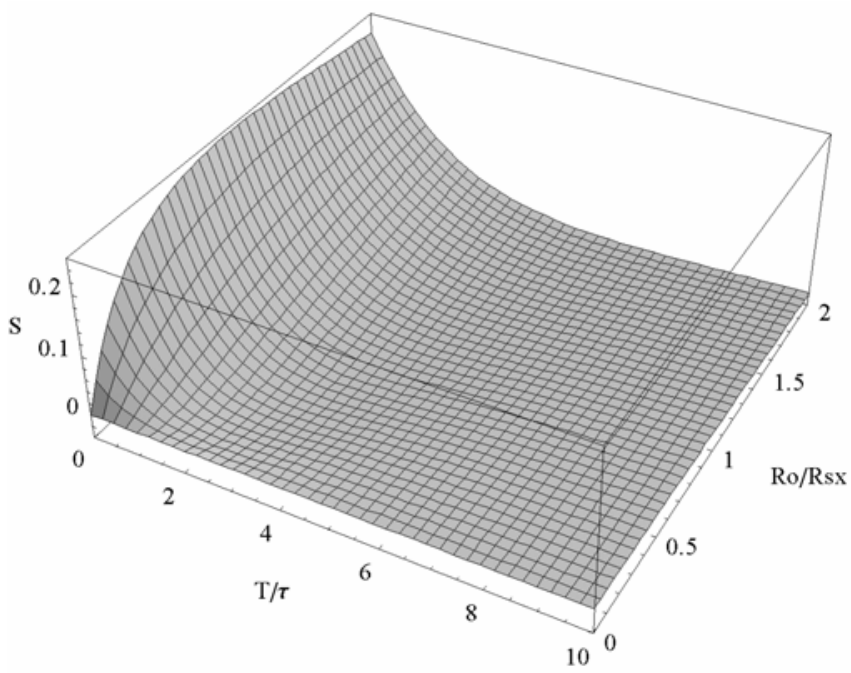

Fig. 9 Surface plot of measurement sensitivity $S$ depending on $T / \tau$ and $R_{0} / R_{S X}$

Dependence of the sensitivity on the relative variation of the sampling instant $\mathrm{d} T / T$ is defined as:

$S_{T}=(d S) /(d T / T)=-\frac{y \cdot x}{(y+1)^{4}} \cdot[2 \cdot(1+y)-x] \cdot e^{-\frac{x}{1+y}}$

and is shown in Fig. 10. The curve $S_{T}$ for a certain $R_{0} / R_{S X}$ passes through zero and for this value of $T / \tau$ the sensitivity curve $S$ has a minimum.

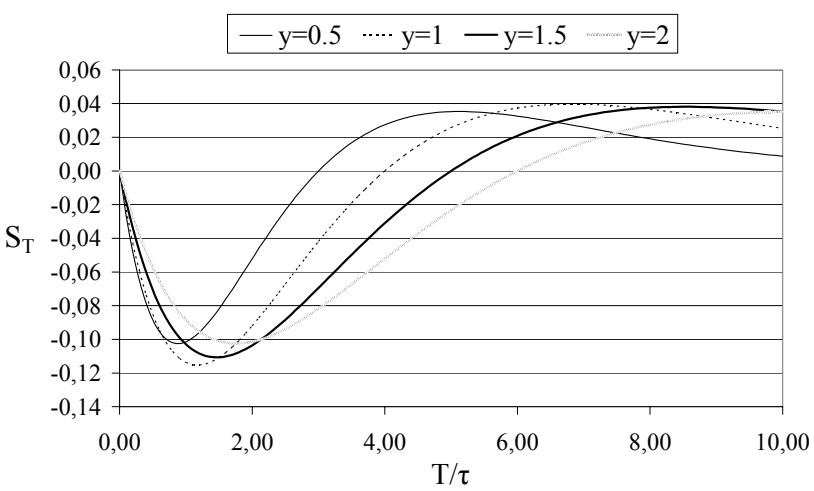

Fig. 10 Dependence of the measurement sensitivity on voltage sampling instant, $S_{T}$ with $y=R_{0} / R_{S X}$ as a parameter
Dependence of the sensitivity on $R_{0} / R_{S X}$ ratio is defined as:

$$
\begin{aligned}
& S_{R}=(d S) /[d y / y]= \\
& =-\frac{y}{(y+1)^{5}} \cdot e^{-\frac{x}{1+y}} . \\
& {\left[y^{3}+y^{2} \cdot(1-3 \cdot x)+y \cdot\left(x^{2}-2 \cdot x-1\right)+x-1\right]}
\end{aligned}
$$

and is shown in Fig. 11. The curve $S_{R}$ for a certain $T / \tau$ passes through zero and for this value $R_{0} / R_{S X}$ the sensitivity curve $S$ has a minimum.



Fig. 11 Dependence of the measurement sensitivity on $R_{0} / R_{S X}$ ratio, $S_{R}$ with $x=T / \tau$ as a parameter

\section{CONCLUSION}

The measurement reliability can be improved in view of maximizing the measurement sensitivity and minimizing the sensitivity variations regarding the variations of the sampling instant, $T$ and the variations of the current sampling resistance to the bio-tissue resistance ratio, $R_{0} / R_{S X}$. The positive sensitivity for a small $T / \tau$ ratio is considerably greater than the negative sensitivity but it varies considerably with a change in $T / \tau$ and $R_{0} / R_{S X}$ ratios. For the negative sensitivity $T$ and $R_{0}$ can be chosen so that at the same time the absolute sensitivity is maximum (the minimum of the sensitivity curve) whereby the variations with $T$ and $R_{0}$ are minimized.

\section{REFERENCES}

1. Melinščak M, Šantić A. (2007) Features of voltage pulse plethysmography, The fifth IASTED International Conf. on Biomedical Engineering, Innsbruck (accepted for presentation) 
2. Šantić A, Kovačić D, Bilas V. (1999) Some new aspects of electrical impedance and pulse plethysmography, European Medical and Biological Engineering Conf. EMBEC, Vienna, pp. 114-115

3. Franks W, Schenker W, Schmutz P et al. (2005) Impedance characterization and modeling of electrodes for biomedical applications, IEEE Trans Biomed Eng 52(7):1295-1302

4. Klyuev AL, Rotenbergb ZA, Batrakov VV. (2005) Impedance of a passive iron electrode in a solution containing a reducing agent, Russ J Electrochem 41(1):87-90

5. Geddes LA. (1972) Electrodes and the measurement of bioelectric events. John Wiley \& Sons

6. Šantić A. (1990) Pulse plethysmography in the blood pressure measurement at the finger, $6^{\text {th }}$ IMEKO Conf. on Measurement in Clinical Medicine, Sopron, pp. 29-31

7. Šantić A, Štritof T, Bilas V. (1998) Plethysmography measurements using short current pulses with low duty-cycle, $20^{\text {th }}$ Ann. Int. Conf. of the IEEE EMBS, Hong Kong, pp. 1889-1892

Address of the corresponding author:

Author: Martina Melinscak

Institute: Polytechnic of Karlovac

Street: Ivan Mestrovic 10

City: Karlovac

Country: Croatia

Email: martina.melinscak@vuka.hr 